\title{
Dynamic behaviour of an electrolyser with a two phase solid-liquid electrolyte Part I: Spectral analysis of potential fluctuations
}

\author{
C. GABRIELLI, F. HUET, R. WIART, J. ZOPPAS-FERREIRA* \\ UPR15 du CNRS "Physique des Liquides et Electrochimie", Université Pierre et Marie Curie, Tour 22, \\ 4 place Jussieu, 75252 Paris, Cedex 05, France
}

Received 17 December 1993; revised 13 April 1994

The dynamic behaviour of a simplified electrolyser with a two phase solid-liquid electrolyte was investigated. The current-voltage curve and the impedance of the working electrode (current collector) was measured. In addition the fluctuations of the working electrode potential and of the electrolyte resistance close to the electrode were analysed, especially by spectral analysis. Conductive zinc-coated polystyrene beads and insulating glass beads were compared. For glass beads the kinetics were controlled by ohmic drop fluctuations due to the collisions of the beads onto the collector. With zinc beads the charge exchanges between the beads and the current collector were also observed and contributed to the potential fluctuations. A complementary analysis of the elementary potential and electrolyte resistance transients will be reported in Part II; the electrochemical behaviour of the collector will then be modelled.

\section{Introduction}

Electrical vehicles need batteries with sufficient energy and power densities, low toxicity, low temperature dependence, low investment and easy maintenance. In spite of the good properties of zinc as an anode for batteries (high specific capacity, high exchange current density, low cost, easy use of the electrodes and low toxicity) several difficulties limit zinc cycling in rechargeable batteries. The main drawbacks are related to $[1,2]$ :

(i) Continuous change of the electrode geometry (shape change) during charge-discharge cycling which leads to a progressive decrease in the system capacity.

(ii) Non adhesion of spongy deposits and dendritic growth of zinc during the battery recharge leading to internal short-circuits.

(iii) Tendency of zinc to corrode in concentrated $\mathrm{KOH}$ medium giving zincate ions, which leads to a self-discharge and a decrease in the storage capability of the battery.

To avoid the main drawbacks of zinc electrodes, a circulating electrolyte and a particulate zinc electrode were used [3]. This device has various advantages, in particular a larger reactional area and increased mass transport. The optimization of this type of battery depends on the hydrodynamic conditions (velocity of the fluid circulation, circulation time), on the cell geometry, on the physicochemical properties of the electrolyte and, also, on the electrochemical reactions involved [4]. It was shown that the use of some addi- tives allows the discharge capacity of the circulating zinc particles/air systems to be increased [5-7].

By using such a cell with zinc particles, the shape change and the dendritic growth of zinc deposits are avoided. Battery recharging can be achieved mechanically: the residual particles and electrolyte are removed at the end of the discharge and replaced by fresh particles and electrolyte. Then the used particles and electrolyte are ex-situ regenerated in fluidized bed electrolysers. Fundamental investigations were carried out on fluidized beds of zinc particles in order to understand the interactions between the moving particles [8-12]. From both impedance measurements and spectral analysis of the potential fluctuations, the time constant of the discharge of the particles previously charged during the collisions with the current feeder was determined. Moreover, it was shown that only continuous chains of particles from the current feeder to the probe carried charge in this electrolyser.

A second alternative consists in recharging the battery in situ in the electrolyser with circulating particles and electrolyte. The hydrodynamics of such a circulating bed electrolyser has been investigated $[13,14]$. By using a redox reaction the mass transport coefficients were measured and showed that, for a permanent circulating regime of conducting particles, only $20 \%$ of the particles are electrochemically active, whatever the flow rate. When a pulsed mode is used, during the flow-off regime, the conducting particles form a fixed bed on the current feeder and are then more electrochemically active. The study of the 
influence of the experimental conditions on the mass transport in this type of bed showed the difficulty of achieving an efficient discontinuous circulation in practical situations. In addition there is a lack of knowledge on the working principles of the circulating particle electrolyser during zinc deposition and dissolution. In particular, the influence of the particle/current feeder collisions on the electrochemical kinetics requires investigation.

These difficulties led to the design of a simpler electrolyser where the main parameters influencing the particle/current feeder collisions are well defined. Thus events involved in these collisions could be analyzed to obtain information on the electrochemical processes taking place at the working electrode. To separate the charge transfer processes from the ohmic drop perturbations by the particle/ collector collisions, both glass beads and zinc beads were used as circulating particles.

In this first paper, the experimental results obtained by impedance spectroscopy and spectral analysis of the fluctuations in practical situations, i.e. for high bead concentration, are presented. A second paper [15] will be devoted to a time analysis of the elementary events, which can be separated for low bead concentrations, thus allowing the electrochemical behaviour of the working electrode to be modelled.

\section{Experimental details}

\subsection{Electrolyser}

The electrolyser is depicted in Fig. 1. In a cylindrical channel (3 $\mathrm{mm}$ diam.) a zinc rod ( $2 \mathrm{~mm}$ diam., Johnson Matthey $99.999 \%$ ) was inserted perpendicularly to the electrolyte flow. The lateral surface of the rod was first insulated by a cataphoretic paint, then its cross section was mechanically polished up to 1200 emery paper grade so that the working electrode was a $0.05 \mathrm{~cm}^{2}$ ellipse whose plane was inclined at $45^{\circ}$ to the electrolyte flow. The counter electrode was a platinum grid covered by a nylon cloth to avoid counter electrode/particle contacts. A luggin capillary allowed the potential to be measured with

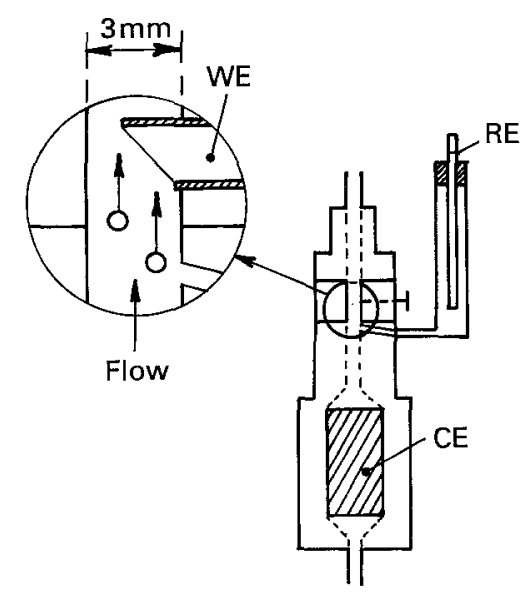

Fig. 1. Electrolyser with two phase solid-liquid electrolyte. WE working electrode; RE reference electrode; CE counter electrode.
Table 1. Physical characteristics of the beads

\begin{tabular}{lll}
\hline Beads & Diameter $/ \mu \mathrm{m}$ & Density $/ \mathrm{g} \mathrm{cm}^{-3}$ \\
\hline glass & $290-320$ & 2.7 \\
zinc & $300-500$ & 2.8 \\
\hline
\end{tabular}

respect to a saturated calomel electrode. The particle-electrolyte mixture was contained in a tank and the circulation was imposed by means of a centrifugal pump. An electromagnetic flowmeter (Picomag DMI 6530) allowed the electrolyte flow, $\nu$, to be measured. Experimental results were obtained in laminar ( $\nu=0.7$ and $1.2 \mathrm{~m} \mathrm{~s}^{-1}$, corresponding to Reynolds numbers 875 and 1500 , respectively) and turbulent $\left(\nu=2.2 \mathrm{~m} \mathrm{~s}^{-1}\right.$ corresponding to Reynolds number 2750) flow conditions. All the experiments were carried out at ambient temperature without electrolyte degassing.

\subsection{Electrolyte and particles}

The electrolyte was made of $\mathrm{KOH}$ solution supersaturated with $\mathrm{ZnO}$ and stabilized by potassium silicate $(8 \mathrm{M} \mathrm{KOH}+1.8 \mathrm{M} \mathrm{ZnO}+1 \%$ silicate). Two types of particles were used: insulating spherical glass beads and zinc beads (polystyrene spheres plated with $1 \mu \mathrm{m}$ of nickel, $1 \mu \mathrm{m}$ of cadmium and then $50 \mu \mathrm{m}$ of zinc, from Sorapec Company). Table 1 gives the physical characteristics of the beads. The bead concentration in the electrolyte was defined as a volume percentage of the beads with respect to the electrolyte (e.g. $2 \%$ bead concentration corresponded to the addition of $5 \mathrm{~cm}^{3}$ of beads into $250 \mathrm{~cm}^{3}$ of electrolyte).

\subsection{Measurement of the PSD of the fluctuations}

Under constant current, $I$, polarization, potential fluctuations, $\Delta V(t)$, around the mean value, $V$, of an electrode undergoing particle impacts may have several causes. The main cause is the perturbation of the primary current lines by particles passing the vicinity of the electrode which give rise to fluctuations of electrolyte resistance, $\Delta R_{\mathrm{e}}(t)$, around the mean value, $R_{\mathrm{e}}$, and, therefore, to fluctuations of the ohmic drop, $\Delta R_{\mathrm{e}}(t) I$. Perturbations of the collector charge, of the electrochemical reaction intermediate concentration also induce fluctuations in the electrode faradaic potential, $\Delta E(t)$. Therefore, the potential difference between the working electrode and the reference electrode fluctuates about the mean value, $V$, such that

$$
\Delta V(t)=I \Delta R_{\mathrm{e}}(t)+\Delta E(t)
$$

The scheme of the experimental arrangement which allowed simultaneous measurement of the fluctuations $\Delta V(t)$ and $\Delta R_{\mathrm{e}}(t)$ has been given in [16]. The electrode faradaic potential fluctuations $\Delta E(t)$ can then be deduced. As shown in [16], the fluctuations in the output signal $\Delta v_{\operatorname{Re}}(t)$ of the electrolyte 
resistance channel was

$$
\Delta v_{\mathrm{Re}}(t)=a \Delta R_{\mathrm{e}}(t)
$$

where $a$ is the sensitivity of the measurement channel $\left(a=19 \mathrm{mV} \Omega^{-1}\right)$.

This arrangement allowed $\Delta R_{\mathrm{e}}=5 \mathrm{~m} \Omega$ peak to peak fluctuations to be detected which was rather low compared with the $R_{\mathrm{e}}=20 \Omega$ mean value. The simultaneous measurements of the $V$ and $R_{\mathrm{e}}$ PSDs were performed in the d.c. $5 \mathrm{kHz}$ frequency range, using a 5451 Hewlett-Packard Fourier analyser.

\section{Experimental results}

\subsection{Current-voltage curve}

Figure 2 gives the current-voltage curve of the zinc electrode under galvanostatic control at an electrolyte velocity of $1.2 \mathrm{~m} \mathrm{~s}^{-1}$. The potentials were corrected for ohmic drop. This curve was not sensitive to the electrolyte velocity between $0.7 \mathrm{~m} \mathrm{~s}^{-1}$ and $2.2 \mathrm{~m} \mathrm{~s}^{-1}$.

In both cathodic and anodic domains the zinc electrode showed the same behaviour as a rotating disc electrode $[17,18]$. Except for low cathodic polarizations known to lead to spongy deposits [17], all zinc deposits were compact (Fig. 3(a)).

During anodic polarization the electrode surface was observed to become black, showing the presence of oxidation products on the dissolving zinc electrode, similarly to a rotating disc electrode [18].

Addition of glass beads or zinc beads did not change the $I / V$ curve, except after about $10 \mathrm{~min}$ for zinc beads in the deposition domain. Then the cathodic polarization decreased and the electrolyte became turbid due to insoluble dissolution products,

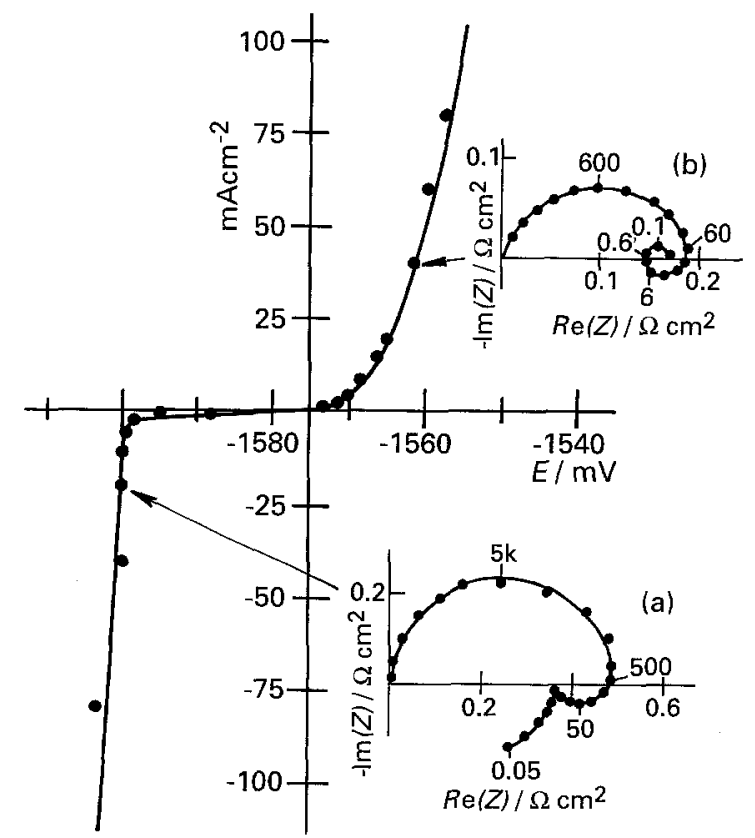

Fig. 2. Current-voltage curve of the zinc electrode in $8 \mathrm{M}$ $\mathrm{KOH}+1.8 \mathrm{M} \mathrm{ZnO}+1 \%$ silicate and impedance diagrams (frequencies in hertz) measured at $20 \mathrm{~mA} \mathrm{~cm}^{-2}$ cathodic and $40 \mathrm{~mA} \mathrm{~cm}^{-2}$ anodic current densities with $2 \%$ glass beads $\left(\nu=1.2 \mathrm{~m} \mathrm{~s}^{-1}\right)$.
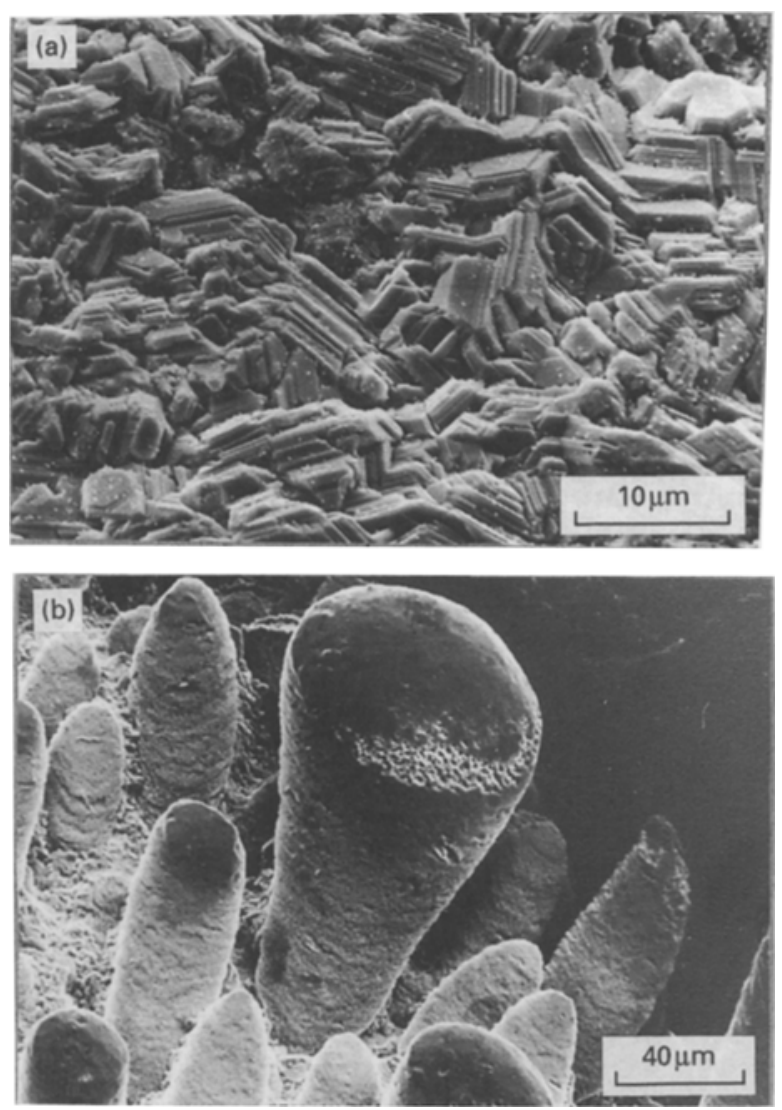

Fig. 3. Morphology of the zinc electrode $\left(\nu=1.2 \mathrm{~m} \mathrm{~s}^{-1}\right)$ : (a) after $1 \mathrm{~h}$ deposition without particles at $10 \mathrm{~mA} \mathrm{~cm}^{-2}$, (b) after $30 \mathrm{~min}$ deposition with $7 \%$ glass beads at $20 \mathrm{~mA} \mathrm{~cm}^{-2}$.

especially $\mathrm{ZnO}$ [19]. As already shown [17], such a polarization decrease was due to anodically dissolved zinc (ADZ) species produced by dissolution of the zinc beads at the rest potential.

The morphology of the zinc deposit was completely modified by the electrode-bead collisions; with glass beads a columnar morphology and an improved brightness appeared (Fig. 3(b)); with zinc beads the formation of a compact deposit was preserved, with smaller grains than in Fig. 3(a), in spite of the presence of $\mathrm{ADZ}$ species in the electrolyte which generally leads to spongy deposits [20].

\subsection{Impedance}

Two typical impedance diagrams are shown in Fig. 2. In the cathodic domain (plot a) a capacitive loop in the high frequency range, related to charge transfer across the double layer, is followed by two inductive loops in the low frequency range. As shown in [17] for a rotating disc electrode, the first corresponds to relaxation of the reaction intermediate $\mathrm{Zn}^{\mathrm{I}}$ concentration whereas the second is induced by relaxation of the thickness of a conducting layer on the electrode. The presence of the beads, either glass or zinc, did not significantly change the impedance except that the mean electrolyte resistance, $R_{\mathrm{e}}$, obtained as the high frequency limit of the impedance, increased with glass beads and decreased with zinc beads. This shows that the beads did not significantly change the 


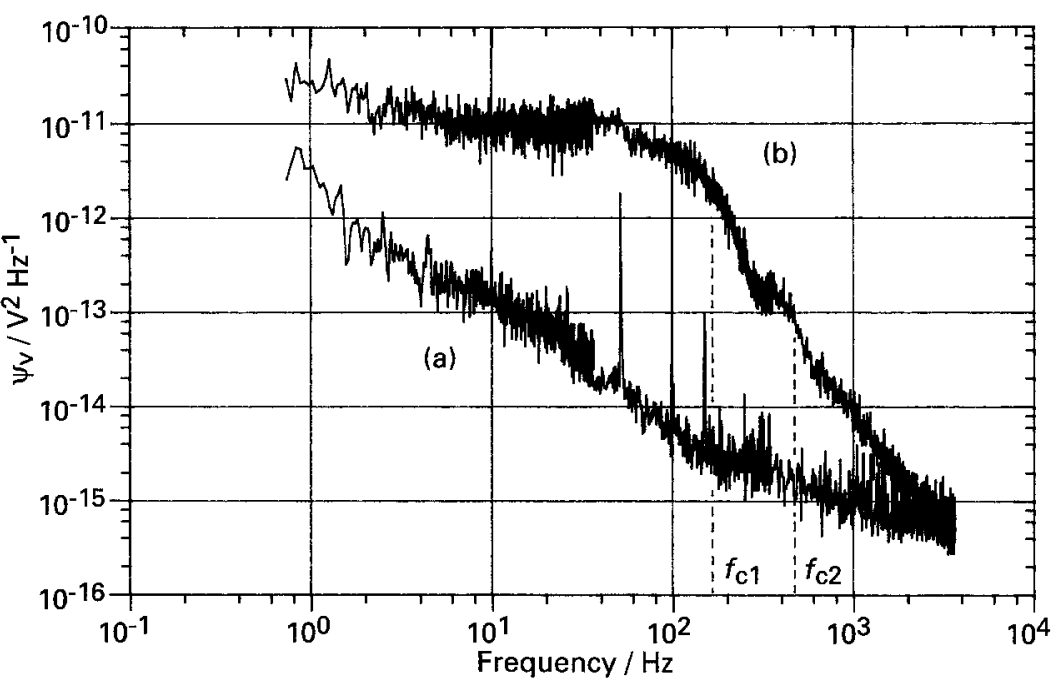

Fig. 4. PSD of the potential fluctuations obtained at a cathodic current density of $10 \mathrm{~mA} \mathrm{~cm}^{-2}\left(\nu=1.2 \mathrm{~m} \mathrm{~s}^{-1}\right)$ : (a) without particles, (b) with $2 \%$ glass beads. kinetics of the electrode processes involved in zinc deposition.

With glass beads in the anodic domain, the impedance (plot (b) in Fig. 2) shows a capacitive loop in the low frequency range. As for a rotating disc electrode [18], this capacitive loop corresponds to the precipitation of dissolution products coupled to the escape of $\mathrm{Zn}^{\mathrm{II}}$ species by diffusion through the pores of a surface layer of oxidation products. The low frequency capacitive loop is cancelled with zinc beads, in agreement with the absence of porous layer on the dissolving zinc electrode.

\subsection{PSD of the potential fluctuations}

3.3.1. Potential fluctuations without particles. Figure 4 (curve (a)) gives the PSD, $\Psi_{\mathrm{v}}$, of the potential fluctuations without particles at a $10 \mathrm{~mA} \mathrm{~cm}^{-2}$ current density in the cathodic domain for the $0.5 \mathrm{~Hz}-10 \mathrm{kHz}$ frequency range. No significant changes were observed when the circulation velocity was changed; however, the PSD was sensitive to electrolyte inhomogeneities (e.g. air microbubbles). This spectrum gave the background noise for this experiment, which could be due to microbubbles in the electrolyte, fluctuations generated by the electrocrystallization and/or the dissolution of the zinc electrode, fluctuations of the electrolyte velocity, etc.

\subsubsection{Potential fluctuations with glass beads. Figure 4} (curve (b)) gives the PSD, $\Psi_{\mathrm{v}}$, of the potential fluctuations for electrolyte with glass beads. A low frequency plateau is followed, after a cut-off frequency, $f_{\mathrm{cl}}$, by a decrease with a more or less constant slope and by a second plateau up to a second cut-off frequency, $f_{\mathrm{c} 2}$. Change in the bead concentration lead to a change in the PSD level without any frequency change. In contrast, an increase in the electrolyte velocity caused an increase in the cut-off frequencies $f_{\mathrm{c} 1}$ and $f_{\mathrm{c} 2}$.

Figure 5 shows that above $20 \mathrm{~mA} \mathrm{~cm}^{-2}$ an increase in cathodic current density leads to an increase in the level of $\Psi_{\mathrm{y}}$ identical in the whole frequency range without modification of the cut-off frequencies. At low polarization current the cut-off frequencies were hardly visible, and the spectrum obtained at zero current was the same as the background noise measured without particles. It was also shown that for current densities larger than $10 \mathrm{~mA} \mathrm{~cm}^{-2}$, the PSD were practically identical in the cathodic and anodic domains. At lower current densities a difference occurred in the low frequency range where the level of $\Psi_{\mathrm{v}}$ was larger in the cathodic range.

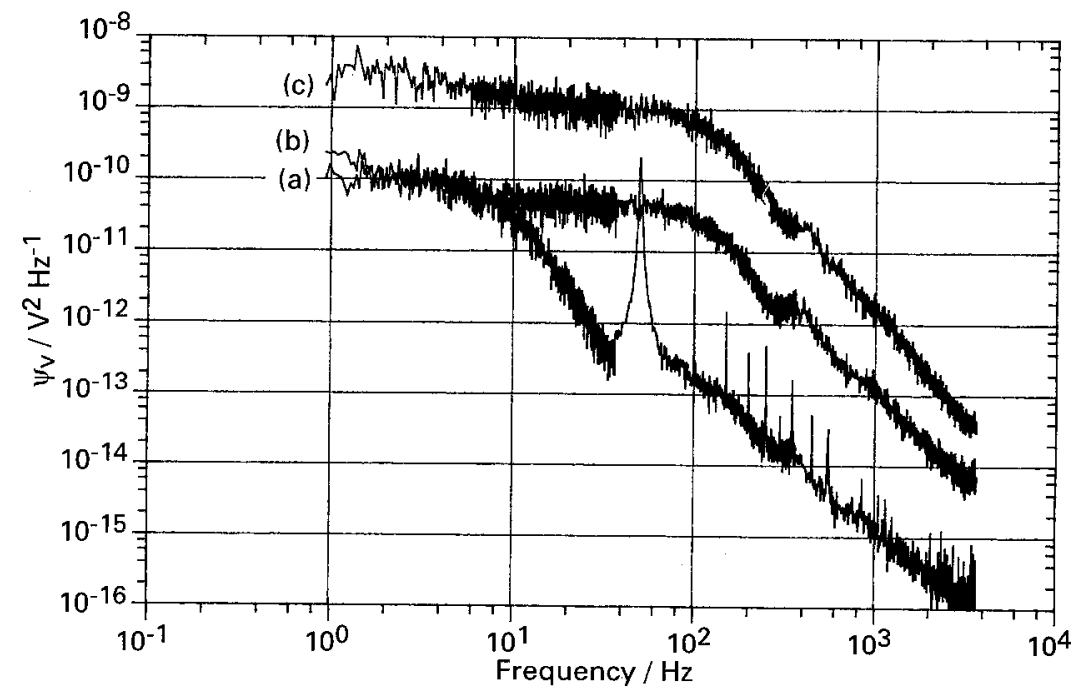

Fig. 5. Influence of the polarization on the PSD of the collector potential fluctuations induced by $2 \%$ glass beads in the cathodic domain $\left(\nu=1.2 \mathrm{~m} \mathrm{~s}^{-1}\right)$ : (a) $1 \mathrm{~mA} \mathrm{~cm}^{-2}$, (b) $20 \mathrm{~mA} \mathrm{~cm}^{-2}$, (c) $120 \mathrm{~mA} \mathrm{~cm}^{-2}$. 


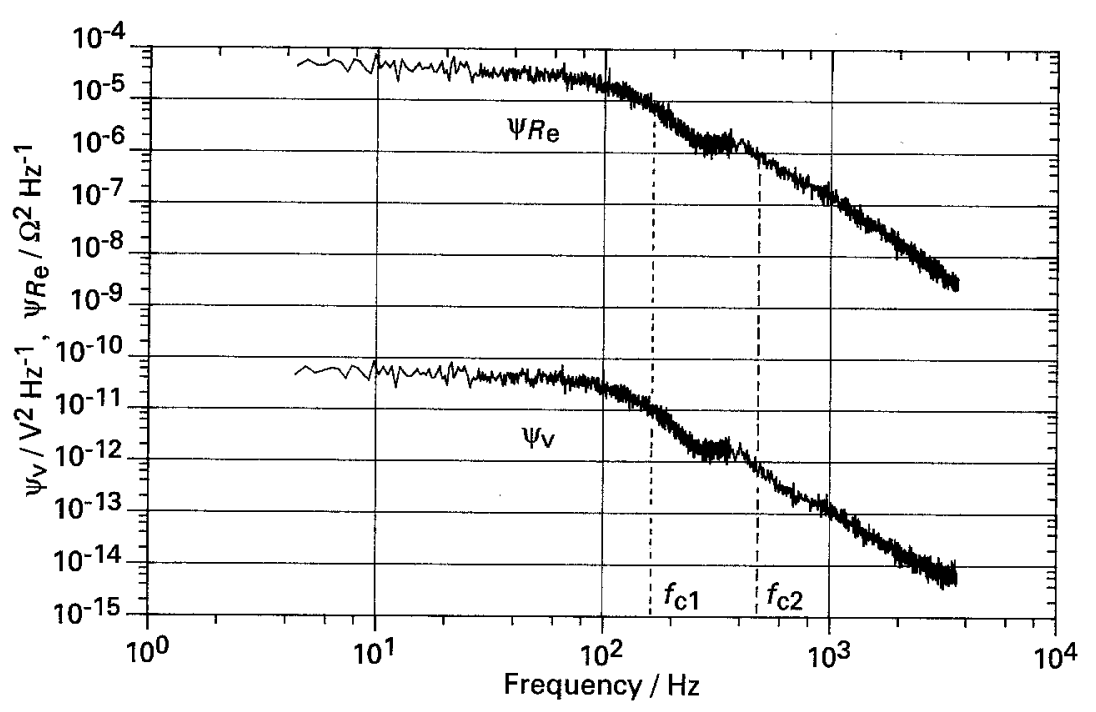

Fig. 6. PSDs $\Psi_{\mathrm{v}}$ of the potential fluctuations and $\Psi_{\mathrm{Re}}$ of the electrolyte resistance fluctuations in presence of glass beads $(2 \%)$ at a $1.2 \mathrm{~m} \mathrm{~s}^{-1}$ electrolyte velocity and a cathodic current density of $20 \mathrm{~mA} \mathrm{~cm}^{-2}$.
To investigate the screening of the electrode surface by the beads, the potential and electrolyte resistance fluctuations were simultaneously measured (Fig. 6). It was noticed that (i) the cut-off frequencies, $f_{\mathrm{cl}}$ and $f_{\mathrm{c} 2}$, appeared on $\Psi_{\mathrm{v}}$ and $\Psi_{\mathrm{Re}}$ and (ii) the level of $\Psi_{\mathrm{Re}}$ are current independent. At a given current, the PSDs $\Psi_{\mathrm{v}}$ and $\Psi_{\mathrm{Re}}$ are parallel in the cathodic and anodic domains. This can be explained by examination of the corresponding time recordings. In Fig. 7 the potential $\Delta V(t)$ and electrolyte resistance $\Delta R_{\mathrm{e}}(t)$ fluctuations induced by glass bead collisions with the current collector are plotted with respect to time. These quantities are plotted with arbitrary origins, both on the time and ordinate scales, as only relative changes are investigated. It is clear that for a high bead concentration $(2 \%)$, the elementary $V$ and $R_{\mathrm{e}}$ transients induced by each bead overlap and cannot be distinguished in Fig. 7. If the $R_{\mathrm{e}}$-time recording is multiplied by the current value $I(I<0$ in the cathodic domain, $I>0$ in the anodic), the

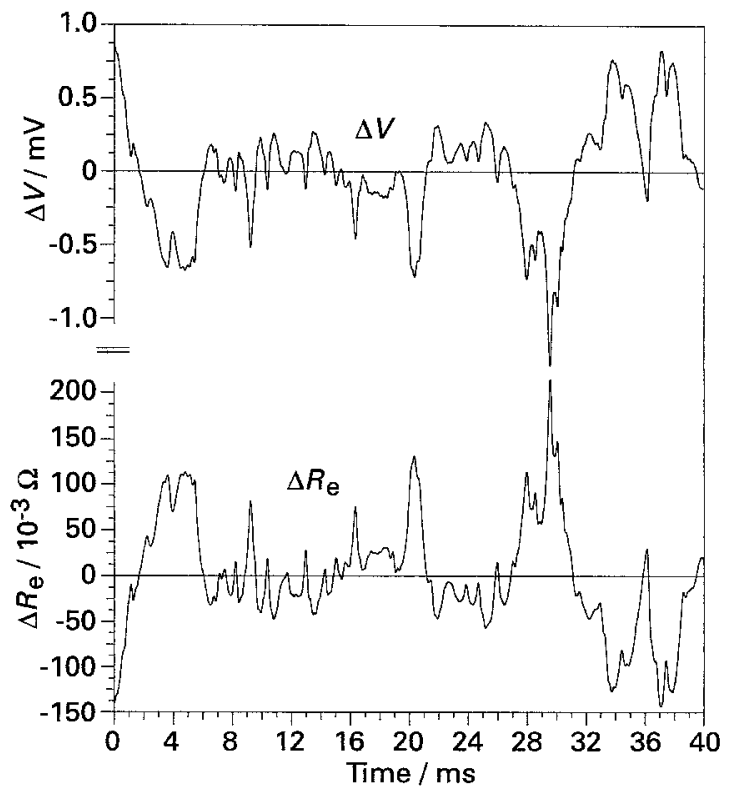

Fig. 7. Time recordings of the potential and electrolyte resistance fluctuations induced by $2 \%$ glass beads at a $120 \mathrm{~mA} \mathrm{~cm}^{-2}$ cathodic current density $\left(\nu=1.2 \mathrm{~m} \mathrm{~s}^{-1}\right)$. Arbitrary origins. ohmic drop-time recording is superposable on the $V$-time recording. The same behaviour was observed in the cathodic and anodic domains for current densities higher than $10 \mathrm{~mA} \mathrm{~cm}^{-2}$. Therefore, since

$$
\Delta V(t)=I \Delta R_{\mathrm{e}}(t)
$$

it can be concluded that for these current densities the fluctuations of the collector potential were explained by the changes in the electrolyte resistance due to the screening of the electrode surface by the beads. This is consistent with the displacement of the cutoff frequencies observed with changing electrolyte velocity. Then, the PSDs are related by the following relationship:

$$
\Psi_{\mathrm{v}}(f)=I^{2} \Psi_{\mathrm{Re}}(f)
$$

as found in Fig. 6, the proportionality factor between the PSDs $\Psi_{\mathrm{v}}$ and $\Psi_{\mathrm{Re}}$ being $I^{2}=10^{-6} \mathrm{~A}^{2}$.

The validity of this relationship increased as the polarization increased (as the ohmic drop became the predominant term) and/or as the bead concentration was higher (as $\Delta R_{\mathrm{e}}$ and the ohmic drop increased with the collision rate). As an example for a higher current, the same plots as in Fig. 6 are given for a cathodic current density of $120 \mathrm{~mA} \mathrm{~cm}^{-2}$ in Fig. 6(a) in [21].

3.3.3. Potential fluctuations with zinc beads. With electrolyte containing zinc beads, it was observed that when the beads collided with the polarized collector, fast potential transients appeared. Time recordings (Fig. 8) showed that these transients had the shape of a steep potential jump followed by an exponential decay with a time constant of about $\tau=50 \mu \mathrm{s}$. For a cathodic polarization, anodic potential jumps were observed, i.e. corresponding to a depolarization process. At the same current in the anodic domain, the potential-time recordings were very similar, except that the potential transients were in the opposite direction.

Figure 9 gives the PSD of the potential fluctuations measured in the cathodic domain for high and low zinc bead concentrations. When time elapsed, it was observed that the PSD amplitude decreased at 


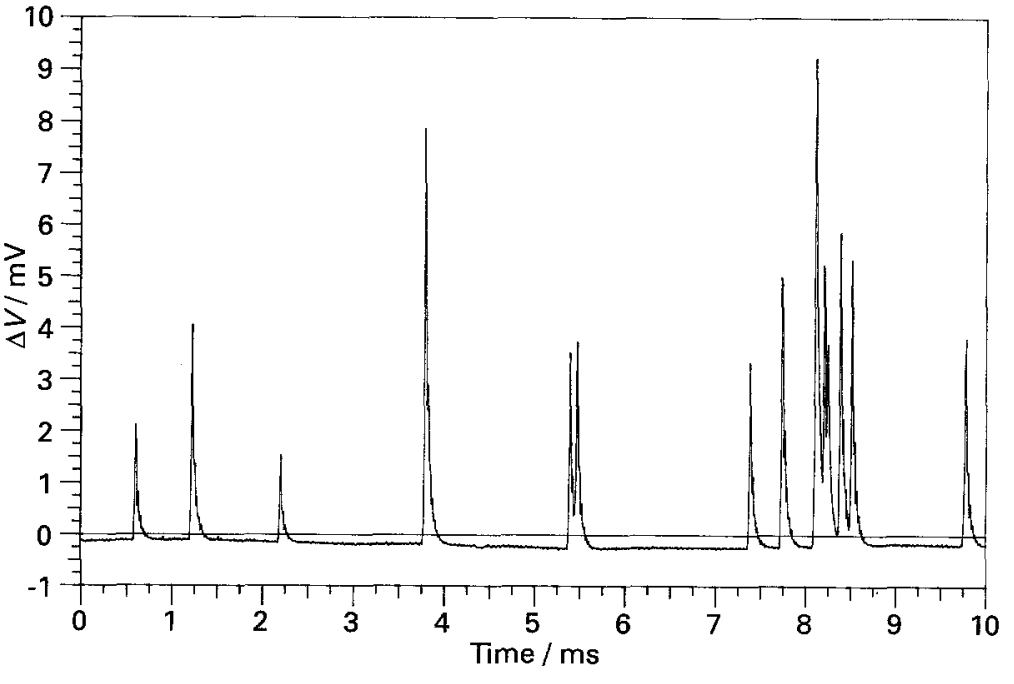

Fig. 8. Time recording of the collector potential fluctuations in presence of zinc beads $(2 \%)$ at a cathodic current density of $10 \mathrm{mAcm}^{-2}(\nu=$ $1.2 \mathrm{~m} \mathrm{~s}^{-1}$ ). Arbitrary origins. high frequencies, and a $170 \mathrm{~Hz}$ cut-off frequency appeared in addition to the $f_{\mathrm{c} 3}=4 \mathrm{kHz}$ cut-off frequency related to the exponential potential decays $\left(f_{\mathrm{c} 3}=1 / 2 \pi \tau\right)$. This behaviour was confirmed with the potential-time recordings: for $i \geqslant 2 \mathrm{~mA} \mathrm{~cm}^{-2}$ the average amplitude of the potential jumps was about $2-4 \mathrm{mV}$ in the first minutes after addition of the beads and decreased down to $0.5 \mathrm{mV}$ after an hour, whereas the time constant of the transients remained identical. In contrast, in the anodic domain the average amplitude of the potential jumps depended more drastically on the polarization (from $0.5 \mathrm{mV}$ at $10 \mathrm{~mA} \mathrm{~cm}^{-2}$ to $3 \mathrm{mV}$ at $120 \mathrm{~mA} \mathrm{~cm}^{-2}$ ) but did not change with time. In the anodic and cathodic domains, the time constant of the potential transients decreased as the polarization increased (from $50 \mu \mathrm{s}$ at $10 \mathrm{~mA} \mathrm{~cm}^{-2}$ to $20 \mu \mathrm{s}$ at $120 \mathrm{~mA} \mathrm{~cm}^{-2}$.

These results showed that the collisions of the conducting beads with the collector induced depolarizing potential fluctuations of the collector. This is consistent with the existence of electrical charge exchanges between the beads which were at equilibrium potential in the electrolyte and the collector which was polarized by means of the galvanostat.
To investigate the influence of the zinc beads on the ohmic drop, the electrolyte resistance fluctuations were also measured. Figure 10 gives the PSDs of the potential $\Psi_{\mathrm{v}}$ and electrolyte resistance $\Psi_{\mathrm{Re}}$ fluctuations, at $20 \mathrm{~mA} \mathrm{~cm}^{-2}$ cathodic current density. For a given current the PSDs measured in the cathodic and anodic domains could be practically superposed. Whatever the current, two cut-off frequencies at $f_{\mathrm{c} 1}=170 \mathrm{~Hz}$ and $f_{\mathrm{c} 2}=500 \mathrm{~Hz}$ were observed on the PSD $\Psi_{\operatorname{Re}}$ as in the case of glass beads. These two cut-off frequencies were more easily detected in $\Psi_{\mathrm{v}}$ at high currents (for example, see Fig. 6(b) in [21] for a current density of $120 \mathrm{~mA} \mathrm{~cm}^{-2}$ ).

Hence the electrode potential fluctuations induced by zinc bead collisions may be explained in the high frequency range $(f>1 \mathrm{kHz})$ by electrical charge exchanges during collisions, and, in the low frequency range $(f<1 \mathrm{kHz})$, by ohmic drop fluctuations. However the PSD $\Psi_{\mathrm{v}}$ could not be obtained from $\Psi_{\mathrm{Re}}$ as easily as for glass beads (Equation 4). The interpretation of the spectrum $\Psi_{\operatorname{Re}}$ measured with zinc beads is complicated; its amplitude is ten times higher than that measured with glass beads, although the mean size and density of the beads were identical.

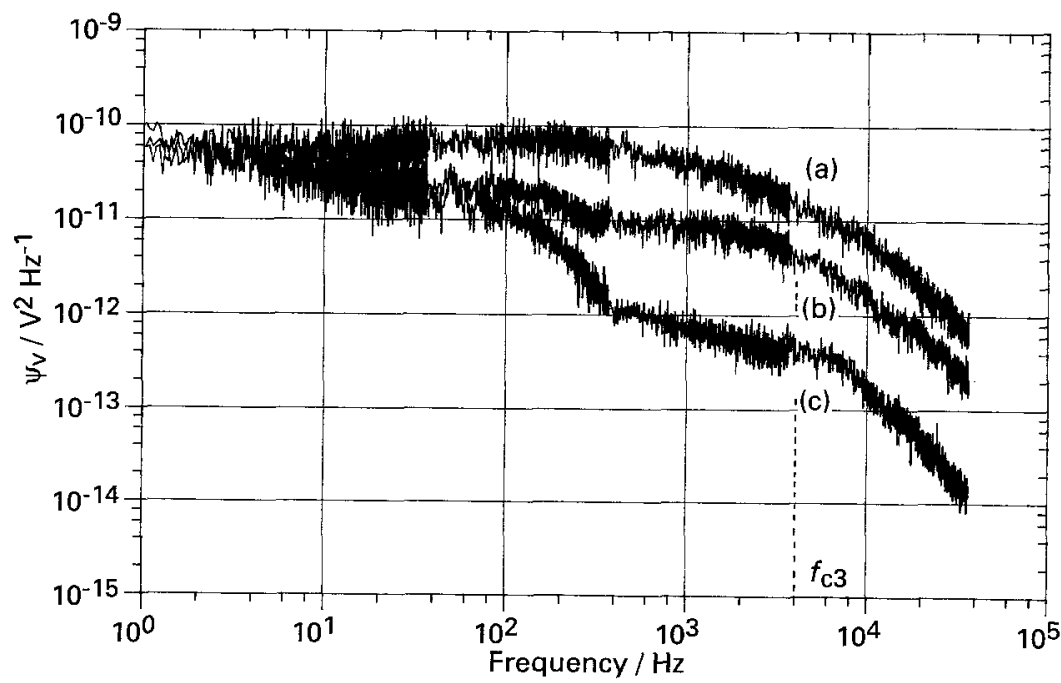

Fig. 9. PSD of the collector potential fluctuations at time $t$ after addition of zinc beads at a cathodic current density of $20 \mathrm{~mA} \mathrm{~cm}^{-2}$ $\left(\nu=1.2 \mathrm{~m} \mathrm{~s}^{-1}\right)$ : (a) $2 \%$ zinc beads, $t<$ $5 \mathrm{~min}$; (b) $0.4 \%$ zinc beads, $t=10 \mathrm{~min}$; (c) $0.4 \%$, zinc beads, $t=3 \mathrm{~h}$. 


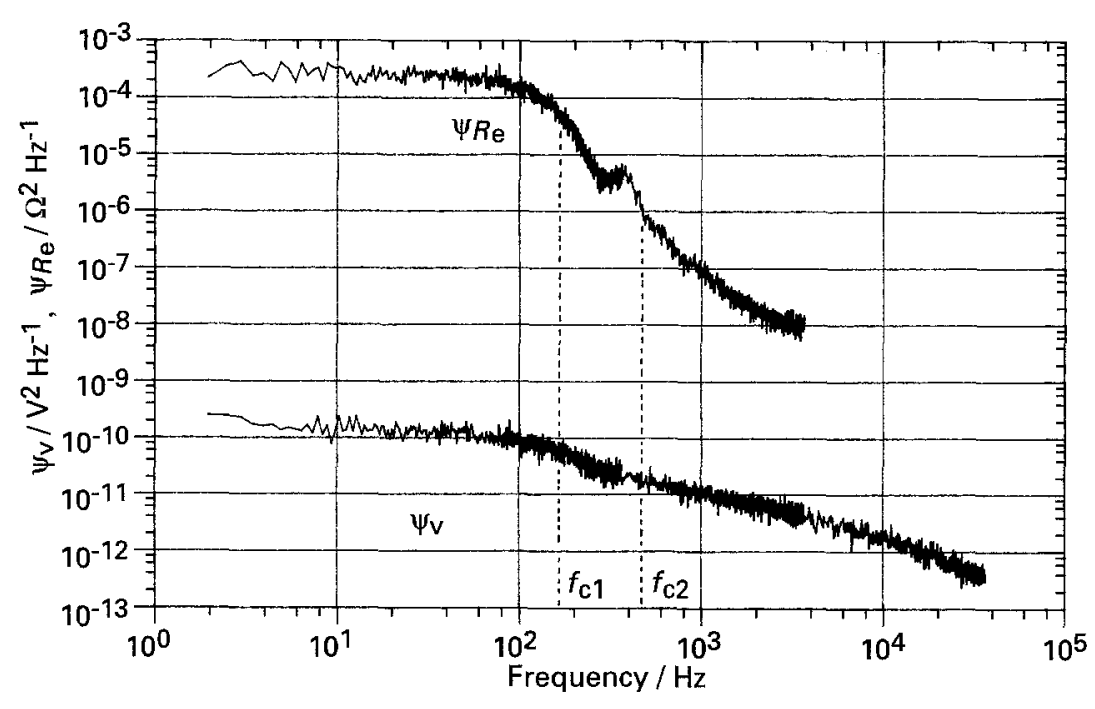

Fig. 10. PSDs $\Psi_{\mathrm{v}}$ of the collector potential fluctuations and $\Psi_{\mathrm{Re}}$ of the electrolyte resistance fluctuations in presence of zinc beads $(2 \%)$ at a $1.2 \mathrm{~m} \mathrm{~s}^{-1}$ electrolyte velocity and a $20 \mathrm{~mA} \mathrm{~cm}^{-2}$ cathodic current density.

\section{Conclusion}

The present study allows the behaviour of a cell with solid-liquid electrolyte to be understood mainly in the high polarization domain $\left(i>10 \mathrm{~mA} \mathrm{~cm}^{-2}\right)$ when insulating glass beads were used. The electrode potential fluctuations were caused by ohmic drop fluctuations due to the passage of the beads close to the current collector. For low polarization other processes occurred. With glass beads, the electrode impedance, which characterizes the time-averaged behaviour of the current collector, was practically unchanged but the collisions appeared to enhance the electrode brightness.

When zinc beads were used the processes involved were not so clear. At any polarization, there was a charge exchange between the zinc beads and the current collector. In the high polarization range there was still an influence of the ohmic drop perturbation due to the presence of the conductive beads but this did not totally explain the potential fluctuations as for insulating beads. The collisions of conductive beads maintained the formation of compact zinc deposit and inhibited the precipitation of dissolution products on the collector.

Due to the large number of particles circulating with the electrolyte, the elementary events could not be separated. Therefore it was impossible to model the processes involved and then the PSD of the collector potential fluctuations. To separate the elementary transients due to each bead, it is necessary to study the fluctuations in the time domain (recordings) and in the frequency domain (PSD) for low bead concentrations. The analysis of these transients will yield a model of the cell behaviour and a way of estimating its characteristic parameters even for high bead concentrations. The beneficial effect of the zinc bead collisions on the deposit structure of the zinc electrode also needs to be investigated. This is the aim of the following paper [15].

\section{References}

[1] J. McBreen and E. J. Cairns, The zinc electrode in "Advances in Electrochemistry and Electrochemical Engineering' (edited by H. Gerischer and C. W. Tobias), John Wiley \& Sons, New York (1978), Vol. 11, pp. 273-352.

[2] J. McBreen, J. Electroanal. Chem. 168 (1984) 415.

[3] G. Bronoel, R. Rouget, N. Tassin and A. Millot, 176th Meeting of Electrochem. Soc., Hollywood, FA (1989), Extended Abstracts no. 1, p. 1.

[4] P. C. Foller, J. Appl. Electrochem. 16 (1986) 527.

[5] H. B. Sierra Alcazar, P. D. Nguyen and A. A. Pinoli, 'Technology Base Research on the Slurry Zinc/Air Battery System', Lawrence Berkeley Laboratory, Subcontract 4543210 (1988).

[6] H. B. Sierra Alcazar and P. D. Nguyen, 22nd Intersociety Energy Conversion Engineering Conference, Philadelphia, PA (1987).

[7] A. Marshall and N. A. Hampson, Electroanal. Chem. Int. Electrochem. 59 (1975) 19.

[8] T. Huh and J. W. Evans, J. Electrochem. Soc. 134 (1987) 308.

[9] Idem, ibid. 134 (1987) 317.

[10] C. Gabriclli, F. Huet, A. Sahar and G. Valentin, J. Appl. Electrochem. 22 (1992) 801

[11] Idem, ibid., 24 (1994) 481.

[12] A. Sahar, $\mathrm{PhD}$ thesis, Universite Paris VI, France (1988).

[13] C. Gabrielli and G. Valentin, A.R.C. no. 3296, PIRSEM, Final Report, Paris (1990).

[14] Z. Chajar, DEA Technical Report, Inst. National Polytechnique de Lorraine, Nancy (1989).

[15] C. Gabrielli, F. Huet, R. Wiart and J. Zoppas-Ferreira, $J$. Appl. Electrochem., in press.

[16] C. Gabrielli, F. Huet and M. Keddam, J. Electrochem. Soc. 138 (1991) L 82

[17] C. Cachet, B. Saidani and R. Wiart, ibid. 138 (1991) 678.

[18] C. Cachet, B. Saidani and R. Wiart, ibid. 139 (1992) 644.

[19] J. W. Evans and G. Savaskan, J. Appl. Electrochem. 21 (1991) 105.

[20] C. Cachet, B. Saidani and R. Wiart, Electrochim. Acta 33 (1988) 405.

[21] C. Gabrielli and F. Huet, J. Appl. Electrochem., 24 (1994) 593. 